\title{
CORRECTION
}

\section{Correction to: Functional virus-specific memory T cells survey glioblastoma}

\author{
Jianfang Ning ${ }^{1} \cdot$ Noah V. Gavil ${ }^{2} \cdot$ Shaoping Wu ${ }^{1}$. Sathi Wijeyesinghe ${ }^{2} \cdot$ Eyob Weyu ${ }^{2} \cdot$ Jun $\mathrm{Ma}^{1} \cdot \mathrm{Ming} \mathrm{Li}^{1}$. \\ Florina-Nicoleta Grigore ${ }^{1}$. Sanjay Dhawan ${ }^{1}$ - Alexander G. J. Skorput ${ }^{3}$. Shawn C. Musial ${ }^{3}$. Clark C. Chen ${ }^{1}$. \\ David Masopust ${ }^{2} \cdot$ Pamela C. Rosato ${ }^{2,3}$ (1)
}

Published online: 18 February 2022

(c) Springer-Verlag GmbH Germany, part of Springer Nature 2022

\section{Correction to: Cancer Immunology, Immunotherapy https://doi.org/10.1007/s00262-021-03125-w}

The original version of this article unfortunately contained a mistake in Figures 1 and 3.
The corrected Figs. 1 and 3 are given in the following page.

The original article has been corrected.

The original article can be found online at https://doi.org/10.1007/ s00262-021-03125-w.

\section{Pamela C. Rosato}

pamela.c.rosato@dartmouth.edu

1 Department of Neurosurgery, University of Minnesota, Minneapolis, MN 55455, USA

2 Center for Immunology, Department of Microbiology and Immunology, University of Minnesota, Minneapolis, MN 55455, USA

3 The Norris Cotton Cancer Center, Department of Microbiology and Immunology, Geisel School of Medicine at Dartmouth College, Lebanon, NH 03756, USA 
A

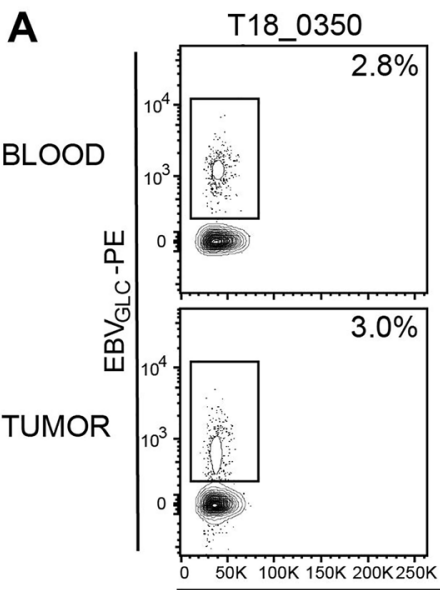

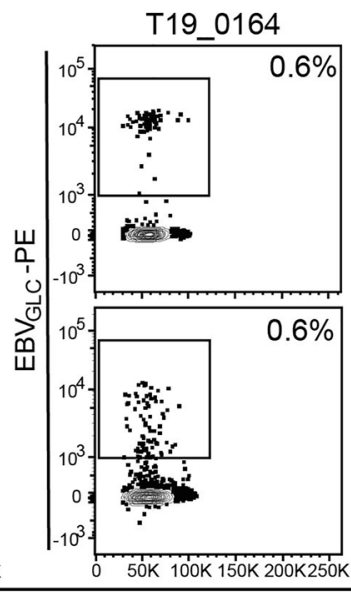

T18_0350

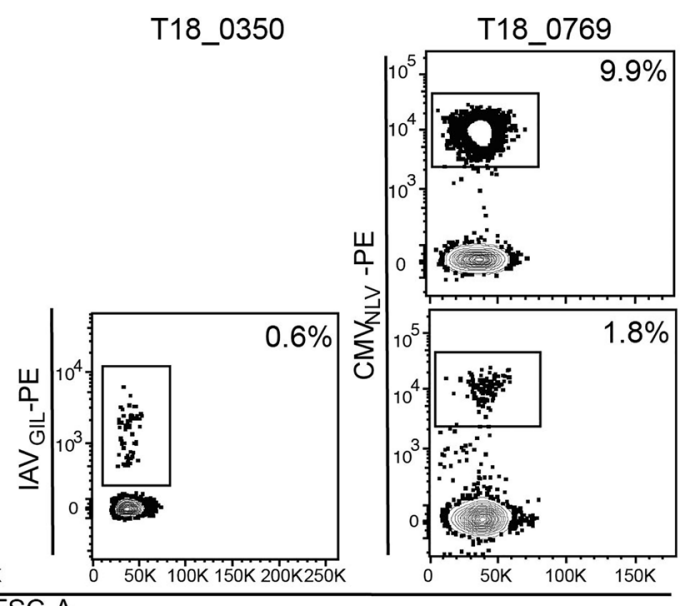

B

$0.1 \%$ tetramer+ $>4$

FSC-A

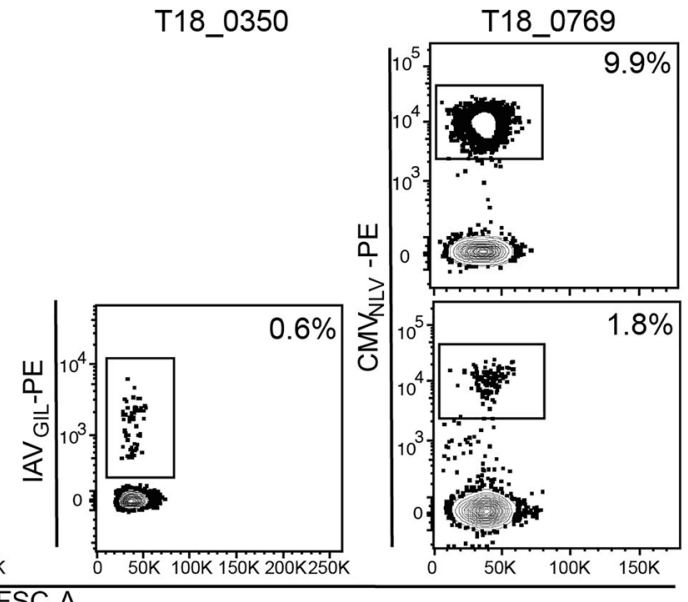

patient ID: T17_1298 T18_0128_T18_0137 T18_0350T18_0358_T18_0566_T18_0769 T18_0929_T18_0969_T19_0158_T19_0164 T19_0175

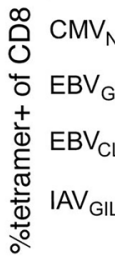

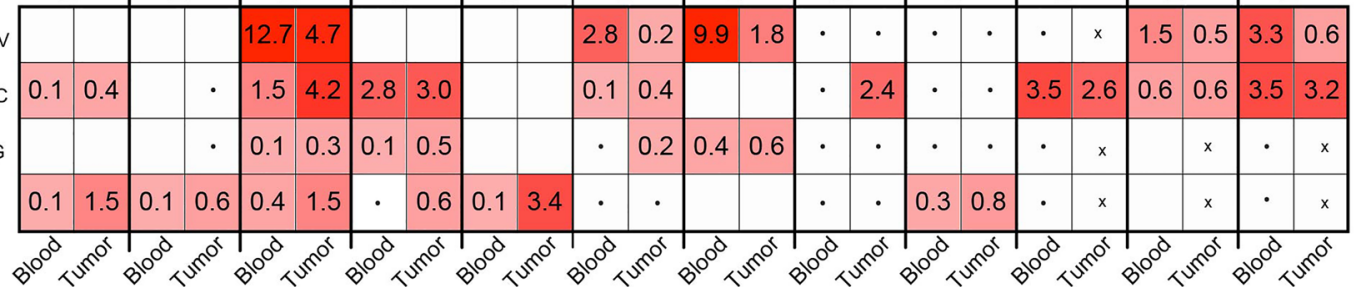

C
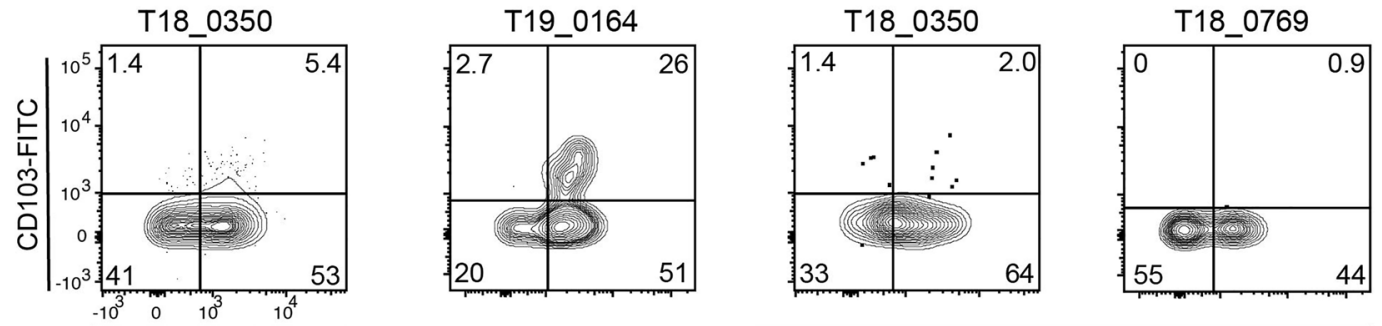

D

$\mathrm{T}_{\mathrm{RM}}$ phenotype of virus-specific $\mathrm{T}$ cells

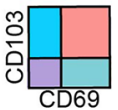

$\square \mathrm{CD} 69-/ \mathrm{CD} 103+\square \mathrm{CD} 69+/ \mathrm{CD} 103+$ $\square$ CD69-/CD103- $\square$ CD69+/CD103-

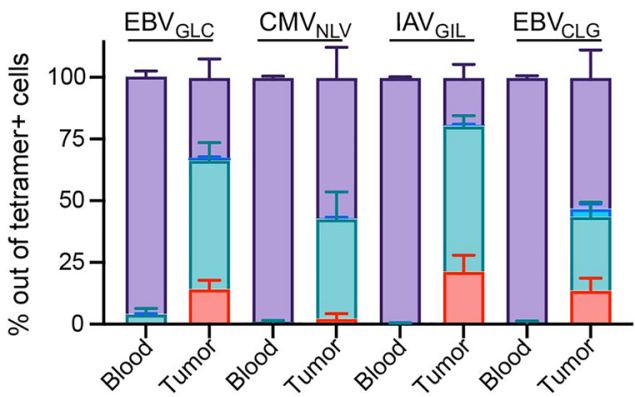

Fig. 1 Virus-specific memory CD8 + T cells populate GBM and have a $\mathrm{T}_{\mathrm{RM}}$ phenotype. a Patient glioblastoma tumors and paired blood stained for HLA-A*02+tetramers specific for EBV $\left(\mathrm{EBV}_{\mathrm{GLC}}\right.$ and $\left.\mathrm{EBV}_{\mathrm{CLG}}\right), \mathrm{CMV}\left(\mathrm{CMV}_{\mathrm{NLV}}\right)$, and Influenza A virus $\left(\mathrm{IAV}_{\mathrm{GIL}}\right)$. Gated on $\mathrm{CD} 8+\mathrm{CD} 3+$ cells. b The frequency (\%) of tetramer-positive populations out of $\mathrm{CD} 8+/ \mathrm{CD} 3+\mathrm{T}$ cells in paired blood and glioblastoma tumors across multiple patients. Dots indicate not enough total CD8 $+\mathrm{T}$ cells were acquired to get an accurate measurement;

\section{CD69- PerCP-Cy5.5}

E T18_0350

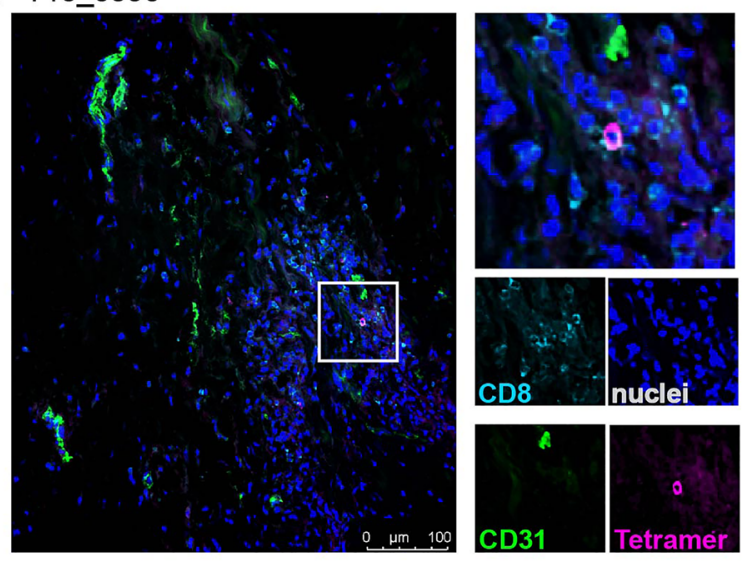

$\mathrm{X}$ indicates data was not collected; Blank indicates tetramer + cells were not detected. c Phenotype of tetramer + cells gated on in (a). d Quantification of CD69/CD103 phenotype of tetramer+cells in all patient populations in blood and paired tumors. e Representative in situ tetramer immunofluorescence staining of glioblastoma tumor. Magenta, EBV/Flu tetramer; Teal, CD8; Blue, 4',6-diamidino2-phenylindole (DAPI)-stained nuclei; Green, CD31. White scale bar $=100 \mu \mathrm{m}$ 

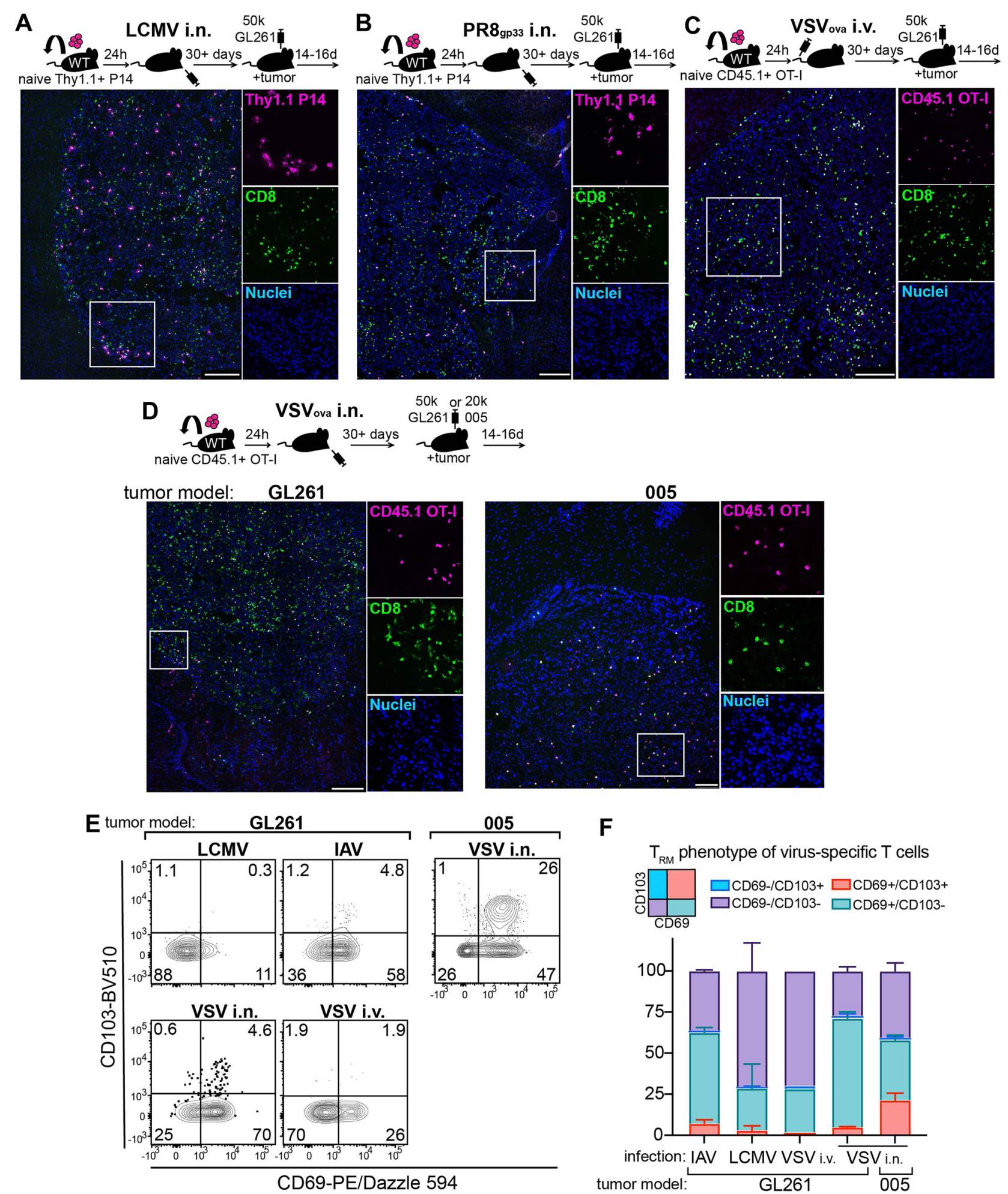

Fig. 3 Establishment of $\mathrm{T}_{\mathrm{RM}}$ phenotype cells in mouse GBM following diverse infections. a-d Schematic of experimental setup utilizing transgenic $\mathrm{T}$ cells specific for antigens expressed by concurrent indicated infection, with corresponding immunofluorescence staining of GBM tumor. Magenta, transgenic T cells (P14 or OT-I); Green, CD8;

Blue, DAPI stained nuclei. Scale bars $=100 \mu \mathrm{m}$. e Representative flow plots gated on transgenic T cells within GBM tumors. f Quantification of E indicating \% of total P14 or OT-I populations expressing CD69/CD103

Publisher's Note Springer Nature remains neutral with regard to jurisdictional claims in published maps and institutional affiliations. 